\title{
Evaluation of simultaneous ${ }^{201} \mathrm{Tl} / 99 \mathrm{mTc}$ dual-isotope cardiac SPECT imaging with model-based crosstalk compensation using canine studies
}

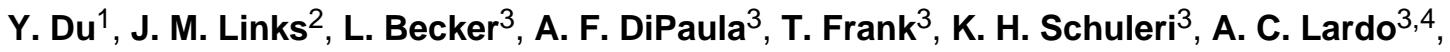 \\ and E. C. Frey ${ }^{1,2,5}$ \\ ${ }^{1}$ The Russell H. Morgan Department of Radiology and Radiological Sciences, School of \\ Medicine, Johns Hopkins University \\ ${ }^{2}$ Environmental Health Sciences, Bloomberg School of Public Health, Johns Hopkins University \\ ${ }^{3}$ Division of Cardiology, Department of Medicine, School of Medicine, Johns Hopkins University \\ ${ }^{4}$ Department of Biomedical Engineering, School of Medicine, Johns Hopkins University \\ ${ }^{5}$ Department of Electrical and Computer Engineering, Whiting School of Engineering, Johns \\ Hopkins University
}

\section{Abstract}

Background-Simultaneous ${ }^{201} \mathrm{Tl} /{ }^{99 \mathrm{~m}} \mathrm{Tc}$-Sestamibi dual-isotope myocardial perfusion SPECT imaging can reduce imaging time and produce perfectly registered rest/stress images. However, crosstalk from ${ }^{99 \mathrm{~m}} \mathrm{Tc}$ into ${ }^{201} \mathrm{Tl}$ images can significantly reduce ${ }^{201} \mathrm{Tl}$ image quality. We have developed a model-based compensation (MBC) method to compensate for this crosstalk. The method has previously been validated with phantom and simulation studies. In this study, we evaluated the MBC method using a canine model.

Methods-Left anterior descending or left circumflex coronary artery stenoses were created in 50 adult mongrel dogs weighing $20-30 \mathrm{~kg}$. The dogs were injected with $111 \mathrm{MBq}(3 \mathrm{mCi})$ of ${ }^{201} \mathrm{Tl}$ at rest, and a SPECT study acquired. Stress was induced by administering adenosine to the dog, followed by injection of $740 \mathrm{MBq}(20 \mathrm{mCi})$ of ${ }^{99 \mathrm{~m}} \mathrm{Tc}$-Sestamibi at peak stress. A second SPECT study was performed with data acquired in both ${ }^{201} \mathrm{Tl}$ and ${ }^{99 \mathrm{~m}} \mathrm{Tc}$ energy windows to provide simultaneous dual-isotope projection data. The images were reconstructed using the ordered subsets-expectation maximization (OS-EM) reconstruction algorithm with compensation for attenuation, scatter and detector response. For simultaneously acquired ${ }^{201} \mathrm{Tl}$ data, we also applied the MBC method to compensate for crosstalk contamination from ${ }^{99 \mathrm{~m}} \mathrm{Tc}$.

\footnotetext{
Disclosure:

Some images in this paper were reconstructed with software licensed by Johns Hopkins University to GE Healthcare, Inc. Under a licensing agreement between GE Healthcare, Inc. and the Johns Hopkins University, Dr. Frey is entitled to a share of royalty received by the University on sales of products that use this software. This arrangement has been reviewed and approved by the Johns Hopkins University in accordance with its conflict of interest policies.

The content of this work is solely the responsibility of the authors and does not necessarily represent the official view of the PHS or its various institutes.
} 
Results-Without compensation, ${ }^{99 \mathrm{~m}} \mathrm{Tc}$ crosstalk increased the estimated ${ }^{201} \mathrm{Tl}$ activity concentration in the rest images and reduced defect contrast. After MBC, the ${ }^{201} \mathrm{Tl}$ images were in good agreement with the registered single isotope images and ex vivo count data. The ischemic (IS) to non-ischemic (NIS) region ${ }^{201} \mathrm{Tl}$ activity concentration ratios were computed for single isotope and dual isotope studies. The correlation with ex vivo IS-NIS ratios was 0.815 after MBC, compared to the 0.495 from data without compensation. In addition, the regression line for the ISNIS ratios with MBC was almost parallel to the line of identity with a slope of 0.93 , compared to a slope of 0.45 without compensation.

Conclusions-These results demonstrate that model-based crosstalk compensation can provide substantial reduction of crosstalk effects in simultaneously acquired myocardial perfusion SPECT images in living biological systems.

\section{Keywords}

Dual-isotope; cardiac SPECT; model-based crosstalk compensation; myocardial perfusion

\section{Introduction}

Myocardial perfusion single-photon emission computed tomography (SPECT, MPS) is a useful tool for detection and characterization of coronary artery disease ${ }^{1-4}$. In general, MPS requires two imaging sessions, one each for the rest and stress images ${ }^{5,67}$. This can make patient scheduling challenging and reduce throughput. In addition, the fact that the stress and rest images are obtained separately means that they may not be perfectly registered, and motion artifacts and artifacts due to anatomical features in the patient such as the diaphragm may be different in the two images. These factors can complicate the use of MPS images to accurately categorize patients.

Dual-isotope simultaneous acquisition (DISA) protocols have been proposed to allow simultaneous imaging of radiopharmaceuticals labeled with different radionuclides ${ }^{2}$. For instance, in cardiac imaging, simultaneous acquisition of ${ }^{201} \mathrm{Tl}$ injected at rest and ${ }^{99 \mathrm{~m}} \mathrm{Tc}-$ Sestamibi injected at stress can be used to acquire rest and stress studies in a single scan ${ }^{8,9}$. In addition to providing perfect registration in time and space, DISA techniques promise to provide reduced acquisition time, improved patient comfort, and better clinical throughput than single isotope or dual isotope techniques using sequential acquisitions. However, for DISA imaging, interactions of the photons in the patient, collimator-detector system, or the limited energy resolution of gamma cameras can result in crosstalk contamination between the two isotopes. This crosstalk results in a reduction in image quality that limits both quantitative accuracy and the ability to detect abnormalities and classify patients based on the simultaneously acquired images. Previous work has shown that, in the case of ${ }^{201} \mathrm{Tl}$ and ${ }^{99 \mathrm{~m}} \mathrm{Tc}-$ Sestamibi rest and stress imaging, crosstalk degrades the diagnostic image quality ${ }^{10}$.

A number of techniques have been proposed to reduce the impact of crosstalk and compensate for its effects. The methods usually involve estimating and subtracting the crosstalk prior to reconstruction or reconstruction-based compensation ${ }^{11-16}$. Reconstructionbased compensation methods use iterative reconstruction algorithms combined with methods 
for estimating the crosstalk contamination either before reconstruction or during reconstruction. Crosstalk estimation can be performed either in the spatial domain using crosstalk models ${ }^{16-18}$ or using energy window based methods ${ }^{11,12,15}$.

We have developed a model-based crosstalk compensation (MBC) method that models photon interactions inside both the object and the collimator-detector system ${ }^{18-20}$ and compensates for this crosstalk via iterative reconstruction ${ }^{21}$. From previous simulation and physical phantom studies, we have demonstrated that MBC methods can provide image quality that is much improved compared to those without compensation, and approaches that obtained with sequential acquisition ${ }^{18,22,23}$. We have also developed dual isotope reconstruction methods that exploit the registration of the rest-stress images to reduce image noise ${ }^{24}$. In this work, we further evaluate the model-based crosstalk compensation using animal studies that provide a test of the methods in a biological system.

\section{Material and Methods}

\section{Animal preparation}

The animal protocol was approved by the Institutional Animal Care and Use Committee (IACUC) of Johns Hopkins University ${ }^{25}$. Fifty adult male mongrel dogs (20-30 kg) were anesthetized, and vital signs, such as blood pressure, pulse-ox, and heart rate were continually monitored. The number of animals was chosen based on our previous experience with the sample size needed to provide enough statistical power to evaluate attenuation and scatter compensation methods. The animals underwent a left thoracotomy, which allowed access to the great vessels and coronary arteries. Aortic, venous, and left atrial catheters were inserted for blood sampling, and radiotracer injection, respectively. The left anterior descending ( $L A D, n=23$ ) or left circumflex ( $L C x, n=27$ ) coronary artery was exposed, and a variable stenosis was induced using an adjustable suture or a plastic screw occluder. An electromagnetic flow probe was temporarily placed around the artery to assess hyperemic response to temporary total occlusion. A degree of stenosis was used that reduced the hyperemic response by approximately $25-75 \%$. The arteries in some animals $(n=6,4 \mathrm{LCx}$ and 2 LAD) were completely occluded to generate acute infarction. The dog's chest was then closed, and the animal transported to the imaging room. The severity of the perfusion defects generated, based on the ischemic to nonischemic area activity concentration ratios obtained from the stress images, ranged from 0.04 (total occlusion) to 0.88 (mild defect). The severities were relatively uniformly distributed over this range. The defect extents ranged from $5 \%$ to $30 \%$ of the total myocardium volume. The size was related to the severity, with lesions with the highest severity also having the largest extent.

\section{Imaging protocol}

All imaging studies were performed on a GE Discovery VH SPECT/CT system. During imaging, the animals were positioned supine on the imaging table and remained in exactly the same position for the duration of the study.

Two imaging protocols were used. In the first protocol, $111 \mathrm{Mbq}(3 \mathrm{mCi})$ of ${ }^{201} \mathrm{Tl}$ was injected. Single isotope rest SPECT and CT projection data were acquired 10-15 minutes 
after thallium injection. After rest imaging, to mimic cardiovascular stress and flow defects, adenosine was administered $(0.28 \mathrm{mg} / \mathrm{kg} / \mathrm{min})$ while the systolic BP was supported by an IV drip of phenylephrine in order to maintain blood pressure above $80 \mathrm{~mm} \mathrm{Hg}$. At peak stress, $740 \mathrm{MBq}(20 \mathrm{mCi})$ of ${ }^{99 \mathrm{~m}} \mathrm{Tc}-$ Sestamibi were injected. The adenosine infusion was stopped 4-5 minutes after ${ }^{99 \mathrm{~m} T c-S e s t a m i b i}$ injection. Simultaneous dual-isotope SPECT imaging was then performed 45 minutes after this to acquire data in both ${ }^{201} \mathrm{Tl}$ and ${ }^{99 \mathrm{~m}} \mathrm{Tc}$ energy windows. The animal was euthanized immediately after acquisition of the SPECT image as described below. For the SPECT acquisition, low energy high resolution (LEHR) collimators were used. Projections were acquired for 28 seconds per view in 60 views over $180^{\circ}$ from $45^{\circ} \mathrm{RAO}$ to $45^{\circ} \mathrm{LPO}$. The projection data were acquired in a $128 \times 128$ image with a pixel size of $0.345 \mathrm{~cm}$. The energy windows were $20 \%$ wide and centered at 140.5 $\mathrm{keV}$ for ${ }^{99 \mathrm{~m}} \mathrm{Tc}$; windows of $30 \%$ width centered at $72 \mathrm{keV}$ and $15 \%$ centered at $167 \mathrm{keV}$ were used for ${ }^{201} \mathrm{Tl}$. After each SPECT scan, low dose CT images were acquired and used to generate attenuation maps for both the ${ }^{99 \mathrm{~m}} \mathrm{Tc}$ window and ${ }^{201} \mathrm{Tl}$ windows. For this imaging protocol, 12 dogs had LAD defects, and 13 had LCx defects. This protocol had the advantage of providing both simultaneous and separately acquired ${ }^{201} \mathrm{Tl}$ projections, and allowed direct comparison of the images. However, there was a long delay ( 3 to 4 hours) between the ${ }^{201} \mathrm{Tl}$ injection and the euthanizing of the animal for ex vivo counting of the myocardium (described below), which could result in redistribution of the ${ }^{201} \mathrm{Tl}^{26}$.

The second protocol was more similar to how the protocol would be implemented clinically, and provided a ${ }^{201} \mathrm{Tl}$ image that more truly represented the rest blood flow. Adenosine was first administered and $740 \mathrm{MBq}(20 \mathrm{mCi})$ of ${ }^{99 \mathrm{~m}} \mathrm{Tc}$-Sestamibi were injected at peak stress. Forty-five minutes after ${ }^{99} \mathrm{~m} \mathrm{Tc}$-Sestamibi injection $111 \mathrm{Mbq}(3 \mathrm{mCi})$ of ${ }^{201} \mathrm{Tl}$ were injected at rest. A dual-isotope SPECT acquisition was performed $10-15$ minutes after ${ }^{201} \mathrm{Tl}$ injection with projection images acquired in both energy windows. For this protocol, there were 11 dogs imaged with LAD defects and 14 dogs imaged with LCx defects. This protocol had the disadvantage that it did not allow direct comparison of separately and simultaneously acquired images.

Results from both imaging protocols were compared with ex vivo data described below.

\section{Post imaging ex vivo processing}

After imaging, a suture was placed around the coronary artery at the site of the occlusion or stenosis ${ }^{25}$. The coronary artery was then tied at the point of stenosis to occlude the vessel. Monastral blue dye (MB $0.5 \mathrm{cc} / \mathrm{kg}$ ) was injected into the left atrium to stain myocardium remote to the stenosed territory. The animal was then euthanized with a saturated solution of potassium chloride, if necessary. The overdose was verified with blood pressure, pulse-ox or ECG. The heart was excised and the right ventricle and great vessels were discarded. The left ventricle was then sliced into five or six $1 \mathrm{~cm}$ thick slice rings perpendicular to the short axis. The rings were incubated in $10 \%$ formalin at room temperature overnight. Later, the rings were rinsed free of formalin and weighed and were each affixed to a styrofoam template demarcated with 16 "rays" emanating from a central point. Pins were placed transmurally around the circumference of the rings at the points where the "rays" bisected the endo- and epi-cardial surfaces of the rings. This produced 16 wedges in all of the rings. 
The LV outline and monastral blue areas were traced on acrylic sheet protectors laid over the rings and also were digitally photographed. The apical aspect of all of rings were used except the apex ring where the basal aspect was used (Figure 1). Each of the 16 wedges was then bisected into endo- and epi-cardial halves. The 32 pieces were weighed and then counted in a scintillation well-counter (Perkin Elmer D5003 Cobra gamma counter) for 2-5 minutes each to record the counts in the ${ }^{99 \mathrm{~m}} \mathrm{Tc}$ window $(124-170 \mathrm{keV})$ and the ${ }^{201} \mathrm{Tl}$ window (58-96 keV), respectively. A spillover matrix measured from standard sources was used to correct the contamination between two isotopes in the measured counts. The efficacy of the spillover correction was validated by comparing the corrected ${ }^{201} \mathrm{Tl}$ counts with those measured 85 hours later ( 14 half lives of $\left.{ }^{99} \mathrm{~m} \mathrm{Tc}\right)$, which were almost identical after decay correction. The counts were then normalized by dividing the counts by the weight of each sample. If the rings passed through the ischemic region the ischemic and normal region could be visually differentiated by the monastral blue stain, with the normal region being stained positively by the dye. The ratios of weight-normalized counts between the center wedges of ischemic region and the center wedges of non-ischemic region were then computed for ${ }^{99 \mathrm{~m}} \mathrm{Tc}$ and ${ }^{201} \mathrm{Tl}$, respectively.

\section{Image reconstruction and crosstalk compensation}

The SPECT images were reconstructed using the iterative ordered-subset expectationmaximization (OS-EM) algorithm. Compensations for attenuation, self-scatter, and the collimator-detector response of peak energy photons for each isotope were all modeled during the iterative reconstruction process. Attenuation was modeled using CT generated attenuation maps, with conversion from CT values to appropriate attenuation values performed using software provided on the scanner. Scatter was modeled during reconstruction in the forward projection process, using the ESSE method ${ }^{21,27,28}$. Distancedependent detector response blurring was modeled using analytical models of the collimator geometric response $\mathrm{e}^{29,30}$. A total of 5 iterations with 12 subsets per iteration was used. The numbers of iterations and subsets used were based on the results of a previous study using simulated data ${ }^{23}$. No post-reconstruction filtering was used, in order to avoid introducing additional partial volume effects.

Because the main emission peaks of ${ }^{201} \mathrm{Tl}$ were below the $140.5 \mathrm{keV}$ peak of ${ }^{99 \mathrm{~m}} \mathrm{Tc}$ and the injected ${ }^{201} \mathrm{Tl}$ activities were lower than that of ${ }^{99 \mathrm{~m}} \mathrm{Tc}$-Sestamibi, crosstalk contamination from ${ }^{201} \mathrm{Tl}$ to the ${ }^{99 \mathrm{~m}} \mathrm{Tc}$ energy windows was very small and was ignored. Thus, the ${ }^{99 \mathrm{~m}} \mathrm{Tc}$ images were first reconstructed from the ${ }^{99 \mathrm{~m}} \mathrm{Tc}$ energy window projections of the simultaneously acquired data and treated as contamination free. The ${ }^{99 \mathrm{~m}} \mathrm{Tc}$ distribution in the reconstructed ${ }^{99 \mathrm{~m}} \mathrm{Tc}$ images was then used as an input to the model-based method to compute the crosstalk contamination in the ${ }^{201} \mathrm{Tl}$ window. The method has been previously described in detail ${ }^{23}$. In the model-based method, the photons scattered inside the object, including those directly scattered into the ${ }^{201} \mathrm{Tl}$ energy window as well as photons scattered into higher energy ranges and subsequently interacting in the collimator-detector system and detected in the ${ }^{201} \mathrm{Tl}$ energy window, were modeled using ESSE techniques ${ }^{19}$. The effects of photon interactions inside the collimator-detector system, including collimator scatter and lead X-rays, and finite energy resolution were modeled using pre-calculated collimatordetector response functions (CDRFs) estimated using Monte Carlo simulations of point 
sources at various distances from the surface of the collimator ${ }^{20}$. The estimated crosstalk was then compensated for by adding the estimates to the estimated projections in the forward projection step during iterative reconstruction of ${ }^{201} \mathrm{Tl}$ images ${ }^{18}$. For comparison, we also reconstructed ${ }^{201} \mathrm{Tl}$ images without crosstalk.

The reconstruction and crosstalk estimation was performed using a single core of a $2.2 \mathrm{GHz}$ AMD Opteron $275 \mathrm{CPU}$ and in a computer with $2 \mathrm{~Gb}$ of memory. It took 1 minute per iteration for OS-EM reconstruction with compensation for attenuation, scatter, and collimator-detector response. It took about 10 minutes to estimate crosstalk using the modelbased method. The total time required to generate all the images was around 20 minutes: 5 minutes to reconstruct the ${ }^{99 \mathrm{~m}} \mathrm{Tc}$ images, 10 minutes for crosstalk estimation, and 5 minutes to reconstruct the ${ }^{201} \mathrm{Tl}$ images.

\section{Data analysis}

For all 50 dogs the SPECT images were compared with ex vivo data. First, the reconstructed SPECT images were re-oriented to generate $15-18$ short-axis slices with a $0.345 \mathrm{~cm}$ slice thickness. The short-axis images were then matched with the ex vivo heart ring slices along the long axis by comparing land marks such as the junction of the right and left ventricles and distance from the apex. The short-axis slices were then summed to give $\sim 1 \mathrm{~cm}$ thick rings that matched the thickness of the ex vivo rings. In the SPECT images, the ischemic regions were identified with a $4 \times 4$ voxel region-of-interest (ROI) placed at the center of the most severe area of ischemia. This ROI size was chosen as a compromise between reducing the effects of partial volume and noise and was based on a previous dog study of perfusion defects. A $4 \times 4$ voxel ROI was also placed on the myocardium in a normal area $180^{\circ}$ opposite the center of the ischemic region. The ROIs were defined in the stress ${ }^{99 \mathrm{~m}} \mathrm{Tc}-$ Sestamibi images because they had better defect contrast than in the rest images. The ROIs were then applied directly to the simultaneously acquired ${ }^{201} \mathrm{Tl}$ images without adjustment because they were naturally registered both in time and space. The mean activity concentrations inside the ROIs were then measured and the activity concentration ratio between the ischemic region and normal (non-ischemic) region was computed. The correlation between the ischemic to non-ischemic ratios obtained from the images and the $e x$ vivo data was calculated. The correlation between the simultaneous dual-isotope and single isotope rest images was also calculated for the first acquisition protocol. In addition, we generated circumferential profiles from the short axis images and compared those with profiles generated from ex vivo counting data.

\section{Results}

Figure 2 shows examples of short axis image slices from ${ }^{99 \mathrm{~m}} \mathrm{Tc}$-Sestamibi stress images reconstructed from simultaneously acquired dual isotope data. The examples demonstrate a severe LCX defect. Circumferential profiles of these images are plotted in Figure 3 as well as the result from ex vivo data. The profiles were normalized to have the same averaged intensity in the septal-anterior region, which, for this dog, had normal uptake. There was a good match between SPECT results and the ex vivo data. The edges of the defect are not as sharp in the SPECT images due to limited spatial resolution. 
In Figure 4, the activity concentration ratios between the ischemic and non-ischemic regions computed from simultaneously acquired ${ }^{99 \mathrm{~m}} \mathrm{Tc}$-Sestamibi stress SPECT data are plotted as a function of values from corresponding ex vivo counting results. The regression line had the equation $y=0.77 x+0.07$ and a correlation coefficient of $r=0.914(p<0.0001)$. There was good correlation between the ratios from the SPECT images and the ratios from ex vivo data, indicating that crosstalk from the ${ }^{201} \mathrm{Tl}$ did not affect the image quality of ${ }^{99 \mathrm{~m}} \mathrm{Tc}$ in simultaneously acquired data.

Figure 5 shows short axis slices of the ${ }^{201} \mathrm{Tl}$ rest images from the same dog study as Figure 2. The images are from single isotope ${ }^{201} \mathrm{Tl}$ images and the simultaneously acquired dual isotope data reconstructed without and with model-based crosstalk compensation.

Circumferential profiles from those images are plotted in Figure 6 and compared with the ex vivo results. The profiles were normalized to have the same average intensity in the septalanterior region, which, for this dog, had normal uptake. Without compensation, the crosstalk greatly reduced the contrast in the defect region, as is seen in both Figures 5 and 6. Modelbased crosstalk compensation removed the effect of the crosstalk and results were in good agreement with those from single isotope images and from ex vivo counting.

Figure 7 shows short axis images of the ${ }^{201} \mathrm{Tl}$ rest images from another dog with an LCx defect. The images are from single isotope ${ }^{201} \mathrm{Tl}$ images and the simultaneously acquired dual isotope data reconstructed without and with model-based crosstalk compensation. Polar maps from those images are also shown at the bottom of Figure 7. Clearly, for this dog, without compensation, the crosstalk from high liver uptake of ${ }^{99 \mathrm{~m}} \mathrm{Tc}$ sestamibi completely obscured the defect in the inferior wall and created an artifactual defect in the anterolateral wall. After model-based crosstalk compensation, the artifactual anterolateral defect disappeared and the inferior wall defect was revealed. The short axis images and polar map with $\mathrm{MBC}$ were in good agreement with those from single isotope images.

In Figure 8, the activity concentration ratios between the ischemic region and the normal (non-ischemic) region computed from simultaneously acquired ${ }^{201} \mathrm{Tl}$ rest SPECT data with and without model-based crosstalk compensation are plotted as a function of ratios from the ex vivo data. Data from all 50 dogs are included in the figure. Points with different colors/ shapes were used to denote the first and the second protocols. Without compensation, defects with an ex vivo ischemic to non-ischemic ratio less than 0.5 had a statistically significant reduction in contrast with a mean difference of $0.26 \pm 0.23$ ( $\mathrm{p}=0.006$ from a paired student $\mathrm{t}$-test). After model-based compensation the ischemic to non-ischemic ratio was significantly improved $(\mathrm{p}=0.006)$ with a mean difference of $-0.12 \pm 0.15$. The regression line had an equation $\mathrm{y}=0.45 \mathrm{x}+0.39$ and a correlation coefficient $\mathrm{r}=0.495(\mathrm{p}=0.001)$ for images reconstructed without crosstalk compensation, and $\mathrm{y}=0.93 \mathrm{x}-0.10$ and a correlation coefficient $\mathrm{r}=0.815(\mathrm{p}<0.0001)$ for images reconstructed with crosstalk compensation. After compensation, the data were in good agreement with the ex vivo results. To compare the difference between the slopes and the correlation coefficients of the two methods, a bootstrap method was used to generate 100 sets of simulated data based on the measured results shown in Figure 8. A paired t-test showed that there was a statistically significant difference between the slopes $(0.45$ vs $0.93, \mathrm{p}<0.0001)$ and the correlation coefficients $(0.495$ vs $0.815, \mathrm{p}<0.0001)$. 
In the first protocol, a separate single-isotope ${ }^{201} \mathrm{Tl}$ rest image was also acquired. In Figure 9 , the activity concentration ratios between the ischemic and non-ischemic regions computed from simultaneously acquired ${ }^{201} \mathrm{Tl}$ rest SPECT data with and without modelbased crosstalk compensation are plotted as a function of the ratios from the rest ${ }^{201} \mathrm{Tl}$ SPECT data for the 25 dogs imaged with the first protocol. The regression lines had the equations $y=0.69 x+0.43$ with a correlation coefficient of $r=0.703(p=0.0062)$ for images without crosstalk compensation, and $y=1.01 x+0.13$ with a correlation coefficient of $r=0.861$ ( $\mathrm{p}=0.0001)$ for images reconstructed with crosstalk compensation. Again, to compare the difference between the slopes and correlation coefficients of the two methods, a bootstrap method was used to generate 100 sets of simulated data based on the measured results shown in Figure 9. The paired t-test showed that there was a statistically significant difference between the slopes $(0.69$ vs $1.01, \mathrm{p}=0.0168)$ and the correlation coefficients ( 0.703 vs $0.861, \mathrm{p}<0.0001)$. After compensation, the simultaneously acquired ${ }^{201} \mathrm{Tl}$ data were in good agreement with the single isotope ${ }^{201} \mathrm{Tl}$ images, which also agreed well with the ex vivo data. Overall, the single isotope images had lower IS-NIS ratios (i.e., higher/ better defect contrast) compared to the dual-isotope images and the ex vivo data. The difference between single isotope images results and the ex vivo results was $-0.24 \pm 0.21$ $(\mathrm{p}=0.000125$, paired $\mathrm{t}$-test). The difference between single isotope images results and the model-based compensated dual-isotope results was $-0.13 \pm 0.18$ ( $\mathrm{p}=0.0045$, paired $\mathrm{t}$-test). One explanation of this is that there was a $3-4$ hour delay between the single-isotope ${ }^{201} \mathrm{Tl}$ imaging and the dual-isotope imaging and euthanizing of the animal. The redistribution of ${ }^{201} \mathrm{Tl}$ during this period could have reduced the lesion contrast, causing increased IS-NIS ratios in the dual-isotope images and the ex vivo data. ${ }^{26}$. As demonstrated in Figure 8, all the data points with ex vivo ${ }^{201} \mathrm{Tl}$ activity concentration ratios between the ischemic and non-ischemic regions that were larger than 1.0 were from the first imaging protocol.

\section{Discussion}

Dual-isotope simultaneous acquisition SPECT imaging is a unique tool for simultaneously assessing stress and rest myocardial perfusion with a single imaging session. It greatly shortens the image acquisition time and provides perfectly registered rest/stress images. However, as demonstrated in this work, the crosstalk between two isotopes can significantly reduce image quality and quantitative accuracy, especially for the images of the radionuclide with the lower emission energies. The crosstalk is caused by photon interactions within the object and the collimator-detector system. For simultaneous dual isotope imaging of ${ }^{201} \mathrm{Tl} / 99 \mathrm{~m} \mathrm{Tc}$, because ${ }^{99 \mathrm{~m}} \mathrm{Tc}$ has a higher emission energy, the effects of ${ }^{201} \mathrm{Tl}$ crosstalk on ${ }^{99 \mathrm{~m}} \mathrm{Tc}$ images are small, as shown in Figures 3 and 4 . On the other hand, the ${ }^{99 \mathrm{~m}} \mathrm{Tc}$ crosstalk has a significant impact on the image quality of ${ }^{201} \mathrm{Tl}$. In this study, ${ }^{99 \mathrm{~m}} \mathrm{Tc}$ crosstalk contributed around $50 \%$ of the total counts recorded in the ${ }^{201} \mathrm{Tl}$ energy window. The two main sources of crosstalk are the multiple Compton scatterings of ${ }^{99 \mathrm{~m}} \mathrm{Tc}$ photons in the object that result in photons with energies that lie in the ${ }^{201} \mathrm{Tl}$ energy window, and the lead X-rays emitted from the collimator after ${ }^{99 \mathrm{~m}} \mathrm{Tc}$ photon interactions ${ }^{20}$. Both factors contribute to crosstalk contamination that has a very broad spatial distribution and that reduces the contrast of the entire images, and especially of myocardial perfusion defects. For example, in Figures 5 and 6, crosstalk from the high liver uptake of ${ }^{99 \mathrm{~m}} \mathrm{Tc}-$ Sestamibi greatly 
increased the image intensity in the inferior wall, reducing the contrast of the inferior wall defect and causing it to appear "normal". In some cases (Figure 7), this could even produce an artifactual lesion in the anterior-lateral wall. Thus, without compensation, the simultaneously acquired ${ }^{201} \mathrm{Tl}$ rest images would suggest a less severe or absent rest perfusion defect, or even an artifactual defect, potentially leading to an inaccurate diagnosis.

To reduce the effects of the crosstalk contamination, accurate compensation is required. The model-based method developed in our lab includes complete modeling of imaging physics, and has been demonstrated to effectively remove the effect of crosstalk in phantom and simulation studies. In this work we have demonstrated this in a biological system, as can be seen in results shown in Figures 5-9. In Figures 5 and 6, with model-based compensation the crosstalk was greatly reduced and the images were close to those from single isotope imaging. The ischemic to non-ischemic ratio was also similar to that from ex vivo counting data. The defect contrasts were improved and closer to the truth. As shown in Figure 8, after compensation, the activity concentration ratios between the ischemic and the non-ischemic regions were in good correlation with the ratios from ex vivo data. The regression line was almost parallel to the line of identity, having a slope of 0.93 , indicating that the $\mathrm{MBC}$ gives a more consistent measure of defect severity compared to the true severity over the range of severities studied. By contrast, for the NC case, the severity of severe defects was underestimated and that of mild defects was overestimated. Figure 9 also shows there was good agreement between the activity concentration ratios computed from crosstalk compensated results and the single isotope data, with a correlation coefficient of 0.861 as opposed to 0.703 for data without crosstalk compensation. All these results demonstrate that model-based crosstalk compensation is effective in reducing the crosstalk in biological systems.

In Figures 8 and 9, some values of the activity concentration ratios between ischemic region and the normal region were greater than 1 for very mild defects. This could be partly due to image noise and the fact that for mild reversible perfusion defects the ${ }^{201} \mathrm{Tl}$ rest images may actually have had normal uptake levels in those regions. ${ }^{201} \mathrm{Tl}$ redistribution could also play a role, as Figures 7 and 8 also show that the single-isotope ${ }^{201}$ Tl IS-NIS ratios were smaller than those measured from dual-isotope ${ }^{201} \mathrm{Tl}$ data, which were again smaller than those measured from ex vivo data.

There were two imaging protocols used in this study. For dogs imaged with the first imaging protocol, there was a long period between the ${ }^{201} \mathrm{Tl}$ injection and dual-isotope imaging (i.e., $\sim 3-4$ hours). The redistribution of ${ }^{201} \mathrm{Tl}$ during this period would be expected to reduce the defect contrast, as demonstrated in Figure 8 where all the data points having an ex vivo ischemic to non-ischemic ratio larger than 1.0 were from protocol 1 . This was also demonstrated by the fact that the single-isotope ${ }^{201} \mathrm{Tl}$ images had lower ischemic to nonischemic ratios as compared with both crosstalk compensated dual-isotope images and the ex vivo data ${ }^{26}$, and these differences were statistically significant. This indicates that the second protocol, where the ${ }^{201} \mathrm{Tl}$ is administered shortly before the image acquisition, would be preferred for clinical imaging. 
Two different occlusion locations were used. There were differences between the LCx defect and the LAD defect in images without crosstalk compensation. As shown in Figures 5-7, crosstalk from high ${ }^{99 \mathrm{~m}} \mathrm{Tc}$ activity concentration in the liver had a large impact on the $\mathrm{LCx}$ defect. The effects on the LAD region were not as large due to its distance from the liver. After model-based crosstalk compensation, the differences between the LAD defect and the LCx defect were small. Overall, the data showed that results from both imaging protocols were very similar in terms of comparing NC with MBC. Therefore the aggregate results that included all the defect locations and imaging protocols were used in Figure 8 in order to improve statistical power.

The injected activities of $111 \mathrm{Mbq}(3 \mathrm{mCi}){ }^{201} \mathrm{Tl}$ and $740 \mathrm{MBq}(20 \mathrm{mCi}){ }^{99 \mathrm{~m}} \mathrm{Tc}$ were chosen to be similar to human studies at the time the study was started. For a typical human study that uses $925 \mathrm{MBq}(25 \mathrm{mCi}){ }^{99 \mathrm{~m}} \mathrm{Tc}$ and $111 \mathrm{MBq}(3 \mathrm{mCi}){ }^{201} \mathrm{Tl}$, count levels of around 32,000 counts/view in the ${ }^{99 \mathrm{~m}} \mathrm{Tc}$ energy window and around 14000 counts/view in the ${ }^{201} \mathrm{Tl}$ energy window were observed. For the activities used in this study, the resulting count levels were around 31000 counts/view in the ${ }^{99 \mathrm{~m}} \mathrm{Tc}$ energy window and around 20000 counts/view in the ${ }^{201} \mathrm{Tl}$ energy window. Thus, the noise levels in these dog data were similar to clinical levels, with the noise in the Tl-201 images being somewhat lower. Validation of the MBC method using simulated data with higher noise levels is under way and has shown good results. From multiple noise realization studies we also found that the model-based estimates approximated a mean of crosstalk and thus the compensation could not remove the randomness in the data contributed by the crosstalk photons ${ }^{22}$.

In the model-based method investigated here, crosstalk is estimated by modeling the image formation process. Only projections recorded in the primary energy windows of the two isotopes are required. An alternative method for estimating crosstalk is using projections acquired in multiple energy windows. Nakamura et al achieved good results in patient studies using a multiple energy windows method developed by Moore et al ${ }^{15,31}$. Energy window based methods generally require the use of a large number (greater than 4) of energy windows, a capability not provided by many cameras and not provided by the camera used in this study. In addition, data in the auxiliary energy windows are often quite noisy, especially when the energy windows are narrow. This noise can result in either additional noise in the compensated data or bias in the crosstalk estimates. The principle advantage of energy-based compensation methods is that they are simple to implement and usually do not increase computational complexity. The results of this study demonstrate the capabilities of model-based compensation methods, and thus the feasibility of simultaneous dual isotope imaging, in a biological system. However, they do not provide information about the relative merit of different crosstalk compensation methods.

In recent years, there has been a trend away from the use of ${ }^{201} \mathrm{Tl}$ due to concerns about patient radiation dose. However, ${ }^{201} \mathrm{Tl}$ remains a useful agent with better perfusion characteristics than available ${ }^{99 \mathrm{~m}} \mathrm{Tc}$ SPECT perfusion agents. When combined with a ${ }^{99 \mathrm{~m}} \mathrm{Tc}$ agent in a simultaneous dual-isotope imaging protocol, there are advantages in reduced imaging time, improved patient comfort, and perfectly registered images. This study demonstrates that, with appropriate crosstalk compensation, the potential benefits of simultaneous dual-isotope imaging can be achieved in biological systems. However, dose 
concerns may reduce the clinical impact of this method. However, there are three factors that may allow reduction of the ${ }^{201} \mathrm{Tl}$ activity to a level where the dose is clinically acceptable.

First, in the studies here the simultaneous dual isotope images were essentially acquired in half the total time to acquire the separate rest and stress images. This total reduction in acquisition time could be traded for reduced $\mathrm{T}^{201}$ activity. For example, the acquisition duration could be increased by $50 \%$ and the dose reduced by a third. This would give the same noise level with a one third reduction in acquisition time compared to a separate acquisition protocol.

Second, there have recently been a number of studies indicating that advanced reconstruction and compensation methods can be used to allow reduced acquisition time or dose with no loss of image quality $24,32,33$. There is every reason to believe that similar gains would be obtained for simultaneous imaging. In addition, novel dual-tracer reconstruction algorithms have been developed that take advantage of the inherent spatial registration of simultaneously acquired data. Simulation studies have shown these methods to provide an advantage in perfusion defect detection compared to conventional reconstruction methods ${ }^{24}$. This improved defect detection could be traded for an additional reduction in radiation dose.

Finally, a number of recent novel cardiac SPECT systems have been developed that provide increased sensitivity ${ }^{34,35}$. There is no reason that the model-based crosstalk compensation method presented here could not be adapted to these systems. The extra sensitivity provided by these systems could be used to reduce the ${ }^{201} \mathrm{Tl}$ dose for this dual isotope protocol just as it has for single isotope ${ }^{201} \mathrm{Tl}$ and ${ }^{99 \mathrm{~m}} \mathrm{Tc}$ single isotope protocols.

\section{Conclusions}

We have evaluated simultaneous ${ }^{201} \mathrm{Tl} / 99 \mathrm{~m} \mathrm{Tc}$ rest/stress cardiac imaging with model-based crosstalk compensation using canine models. We observed that crosstalk from ${ }^{99 \mathrm{~m}} \mathrm{Tc}$ photons to the ${ }^{201} \mathrm{Tl}$ energy window reduced contrast in ${ }^{201} \mathrm{Tl}$ rest images and, some instances, caused an artifactual defect in the anterolateral wall for dogs with LCx defects. Compared with ex vivo data, without compensation, the defect severity of ischemic regions in the rest images was significantly reduced for severe defects (mean difference $=0.26 \pm 0.23$, $\mathrm{p}=0.006$ ). After model-based compensation the defect contrast was substantially improved (mean difference $=-0.12 \pm 0.15)$, and the improvement was statistically significant $(\mathrm{p}=0.006)$. The ischemic to non-ischemic ratios were well correlated $(\mathrm{r}=0.815, \mathrm{p}<0.0001)$ with the $e x$ vivo data, indicating that model-based crosstalk compensation can provide a substantial reduction of crosstalk in the simultaneously acquired data. The slope of the regression line was also significantly improved from 0.45 to 0.93 ( $\mathrm{p}<0.0001$ ). These results demonstrate that model-based crosstalk compensation can provide substantial reduction of crosstalk effects in simultaneously acquired myocardial perfusion SPECT images in living biological systems. 


\section{Acknowledgments}

The authors would like to thank Kai Sun, M.S. for help with the statistical analysis. This work was supported by the Public Health Service grant R01-EB00288.

\section{References}

1. Akutsu Y, Nishimura H, Li H, Huang T, Yamanaka H, Shinozuka A, et al. Sequence in appearance or recovery of ischaemia within an exercise-induced ischaemic territory as analyzed by simultaneous dual-isotope single photon emission tomography. Eur Heart J. 2001; 22:194.

2. Asano H, Sone T, Tsuboi H, Sassa H, Takeshima K, Miyazaki Y, et al. Diagnosis of Right Ventricular Infarction by Overlap Images of Simultaneous Dual Emission Computed-Tomography Using Tc-99M Pyrophosphate and Tl-201. Am J Cardiol. 1993; 71(11):902-8. [PubMed: 8465779]

3. Beller GA. Myocardial Perfusion Imaging with Thallium-201. J Nucl Med. 1994; 35(4):674-80. [PubMed: 8151393]

4. Berman DS, Kiat H, Friedman JD, Wang FP, van TK, Matzer L, et al. Separate acquisition rest thallium-201/stress technetium-99m sestamibi dual-isotope myocardial perfusion single-photon emission computed tomography: a clinical validation study. Journal of the American College of Cardiology. 1993; 22(5):1455-64. [PubMed: 8227805]

5. Ben-Haim S, Gips S, Merdler A, Front A, Tamir A. Myocardial stunning demonstrated with rest and post-stress measurements of left ventricular function using dual-isotope gated myocardial perfusion SPECT. Nucl Med Commun. 2004; 25(7):657-63. [PubMed: 15208492]

6. Berman DS, Kiat H, Train KV, Friedman JD, Wang FP, Germano G. Dual-isotope myocardial perfusion SPECT with rest thallium-201 and stress Tc-99m Sestamibi. Card Clin. 1994; 12(2):26170.

7. Heo J, Wolmer I, Kegel J, Iskandrian AS. Sequential Dual-Isotope Spect Imaging with Tl-201 and Technetium-99M-Sestamibi. J Nucl Med. 1994; 35(4):549-53. [PubMed: 8151373]

8. Kiat H, Benari B, Williams C, Wang FP, Vantrain K, Areeda J, et al. Reproducibility of Quantitative Rest T1-201 Stress Tc99M Sestamibi Dual-Isotope Spect for Assessment of Perfusion Defect Extent and Reversibility. J Nucl Med. 1994; 35(5):103.

9. Kiat H, Germano G, Friedman J, Vantrain K, Silagan G, Wang FP, et al. Comparative Feasibility of Separate or Simultaneous Rest Tl-201 Stress Technetium-99M-Sestamibi Dual-Isotope Myocardial Perfusion Spect. J Nucl Med. 1994; 35(4):542-8. [PubMed: 8151372]

10. Ando H, Fukuyama T, Mitsuoka W, Egashira S, Imamura Y, Masaki H, et al. Influence of downscatter in simultaneously acquired thallium-201/technetium-99m-PYP SPECT. J Nucl Med. 1996; 37(5):781-5. [PubMed: 8965145]

11. Buvat I, Hapdey S, Benali H, Todd-Pokropek A, Di Paola R. Spectral factor analysis for multiisotope imaging in nuclear medicine. Lect Notes Comput Sc. 1999; 1613:442-7.

12. Hannequin $P$, Mas J, Germano G. Photon energy recovery for crosstalk correction in simultaneous Tc-99m/Tl-201 imaging. J Nucl Med. 2000; 41(4):728-36. [PubMed: 10768576]

13. Ichihara T, Ogawa K, Motomura N, Kubo A, Hashimoto S. Compton Scatter Compensation Using the Triple-Energy Window Method for Single-Isotope and Dual-Isotope Spect. J Nucl Med. 1993; 34(12):2216-21. [PubMed: 8254414]

14. Knesaurek K. A New Dual-Isotope Convolution Cross-Talk Correction Method - a Tl-201 Tc-99M Spect Cardiac Phantom Study. Med Phys. 1994; 21(10):1577-83. [PubMed: 7869989]

15. Moore SC, English RJ, Syravanh C, Tow DE, Zimmerman RE, Chan KH, et al. Simultaneous Tc-99M/Tl-201 Imaging Using Energy-Based Estimation of the Spatial Distributions of Contaminant Photons. IEEE Trans Nucl Sci. 1995; 42(4):1189-95.

16. Wells RG, Celler A, Harrop R. Analytical calculations of cross-talk for dual-isotope SPECT projections. IEEE Trans Nucl Sci. 2000; 47(3):1202-9.

17. de Jong HWAM, Beekman FJ. Rapid SPECT simulation of downscatter in non-uniform media. Phys Med Biol. 2001; 46(3):621-35. [PubMed: 11277213] 
18. Kadrmas DJ, Frey EC, Tsui BMW. Simultaneous technetium-99m/thallium-201 SPECT imaging with model-based compensation for cross-contaminating effects. Phys Med Biol. 1999; 44(7): 1843-60. [PubMed: 10442716]

19. Frey, EC.; Tsui, BMW., editors. A new method for modeling the spatially-variant, object shape dependent scatter response function in SPECT. 1996 IEEE Nuclear Science Symposium Conference Record; 1996; Anaheim, Ca: IEEE; p. 1082-6.

20. Wang WT, Frey EC, Tsui BMW, Tocharoenchai C, Baird WH. Parameterization of Pb X-ray contamination in simultaneous Tl-201 and Tc-99m dual-isotope imaging. IEEE Trans Nucl Sci. 2002; 49(3):680-92.

21. Kadrmas DJ, Frey EC, Karimi SS, Tsui BMW. Fast implementations of reconstruction-based scatter compensation in fully 3D SPECT image reconstruction. Phys Med Biol. 1998; 43(4):85773. [PubMed: 9572510]

22. He X, Song XY, Frey EC. Application of Three-Class ROC Analysis to Task-Based Image Quality Assessment of Simultaneous Dual-Isotope Myocardial Perfusion SPECT (MPS). IEEE Trans Med Imag. 2008; 27(11):1556-67.

23. Song X, Frey EC, Wang WT, Du Y, Tsui BMW. Validation and evaluation of model-based crosstalk compensation method in simultaneous Tc-99m stress and Tl-201 rest myocardial perfusion SPECT. IEEE Trans Nucl Sci. 2004; 51(1):72-9.

24. He X, Cheng L, Fessler JA, Frey EC. Regularized Image Reconstruction Algorithms for Dualisotope Myocardial Perfusion SPECT (MPS) Imaging using a Cross-tracer Prior. IEEE Trans Med Imaging. 2011; 30(6):1169-83. [PubMed: 20952334]

25. Madar I, Ravert H, DiPaula A, Du Y, Dannals RF, Becker L. Assessment of severity of coronary artery stenosis in a canine model using the PET agent F-18-fluorobenzyl triphenyl phosphonium: Comparison with Tc-99m-tetrofosmin. J Nucl Med. 2007; 48(6):1021-30. [PubMed: 17504876]

26. Beller GA, Watson DD, Ackell P, Pohost GM. Time Course of Tl-201 Redistribution after Transient Myocardial Ischemia. Circulation. 1980; 61(4):791-7. [PubMed: 7357722]

27. Beekman FJ, Kamphuis C, Frey EC. Scatter compensation methods in 3D iterative SPECT reconstruction: A simulation study. Phys Med Biol. 1997; 42(8):1619-32. [PubMed: 9279910]

28. Zeng GL, Gullberg GT. Unmatched projector/backprojector pairs in an iterative reconstruction algorithm. Medical Imaging, IEEE Transactions on. 2000; 19(5):548-55.

29. Metz CE. The geometric transfer function component for scintillation camera collimators with straight parallel holes. Phys Med Biol. 1980; 25(6):1059-70. [PubMed: 7208618]

30. Zeng GL, Gullberg GT, Tsui BMW, Terry JA. Three-dimensional iterative reconstruction algorithms with attenuation and geometric point response correction. IEEE Trans Nucl Sci. 1991; 38:693-702.

31. Nakamura M, Takeda K, Ichihara T, Motomura N, Shimizu H, Saito Y, et al. Feasibility of simultaneous stress Tc-99m-sestamibi/rest (201)T1 dual-isotope myocardial perfusion SPECT in the detection of coronary artery disease. J Nucl Med. 1999; 40(6):895-903. [PubMed: 10452303]

32. Ali I, Ruddy TD, Almgrahi A, Anstett FG, Wells RG. Half-Time SPECT Myocardial Perfusion Imaging with Attenuation Correction. J Nucl Med. 2009; 50(4):554-62. [PubMed: 19289436]

33. DePuey EG, Bommireddipalli S, Clark J, Leykekhman A, Thompson LB, Friedman M. A comparison of the image quality of full-time myocardial perfusion SPECT vs wide beam reconstruction half-time and half-dose SPECT. Journal of Nuclear Cardiology. 2011; 18(2):27380. [PubMed: 21287370]

34. Kacperski K, Erlandsson K, Ben-Haim S, Hutton BF. Iterative deconvolution of simultaneous Tc-99m and Tl-201 projection data measured on a CdZnTe-based cardiac SPECT scanner. Phys Med Biol. 2011; 56(5):1397-414. [PubMed: 21317483]

35. Hutton BF. Developments in cardiac-specific SPECT imaging. Q J Nucl Med Mol Im. 2012; 56(3): 221-9. 


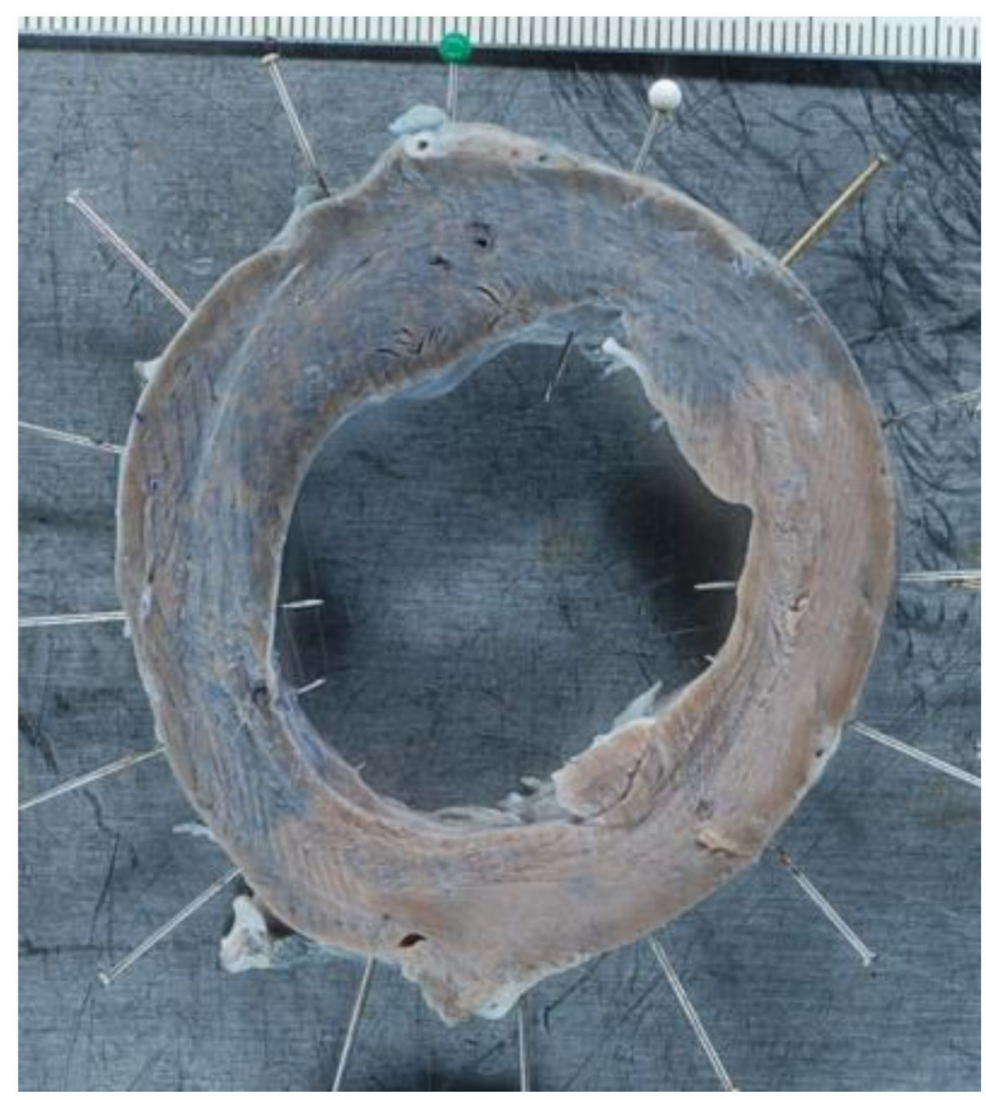

Figure 1.

Example of one ring slice showing the pins used to segment the rings into wedges. The green pin indicates the position of LAD artery and one cut of the first sample for that ring ended at the white pin. The blue stained region had normal perfusion while the unstained region indicates an ischemic area. 


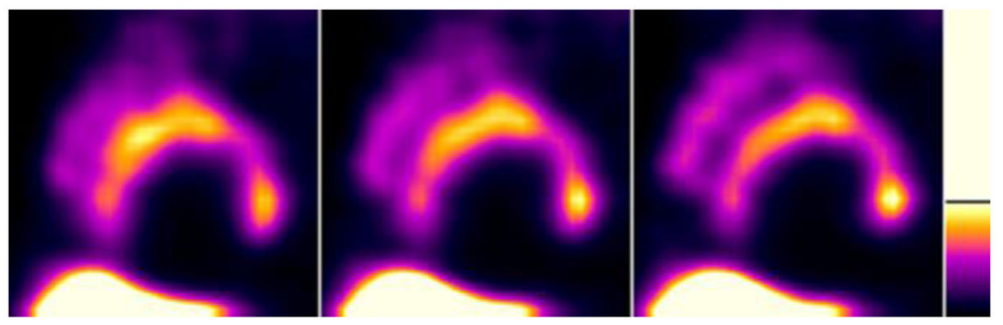

Figure 2.

Three consecutive short axis stress images from a dog having a severe LCX defect. 


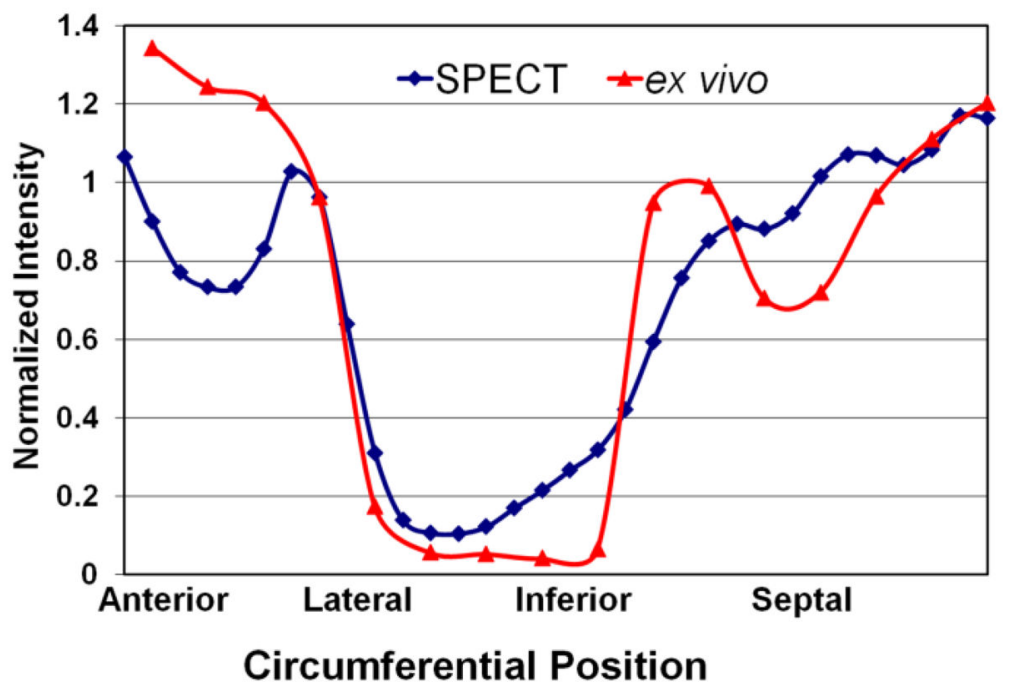

Figure 3.

Circumferential profiles from ${ }^{99 \mathrm{~m}} \mathrm{Tc}$ Sestamibi stress images and ex vivo counting data. The profiles were normalized to have the same averaged intensity in the septal-anterior region, which, for this dog, had normal uptake. 


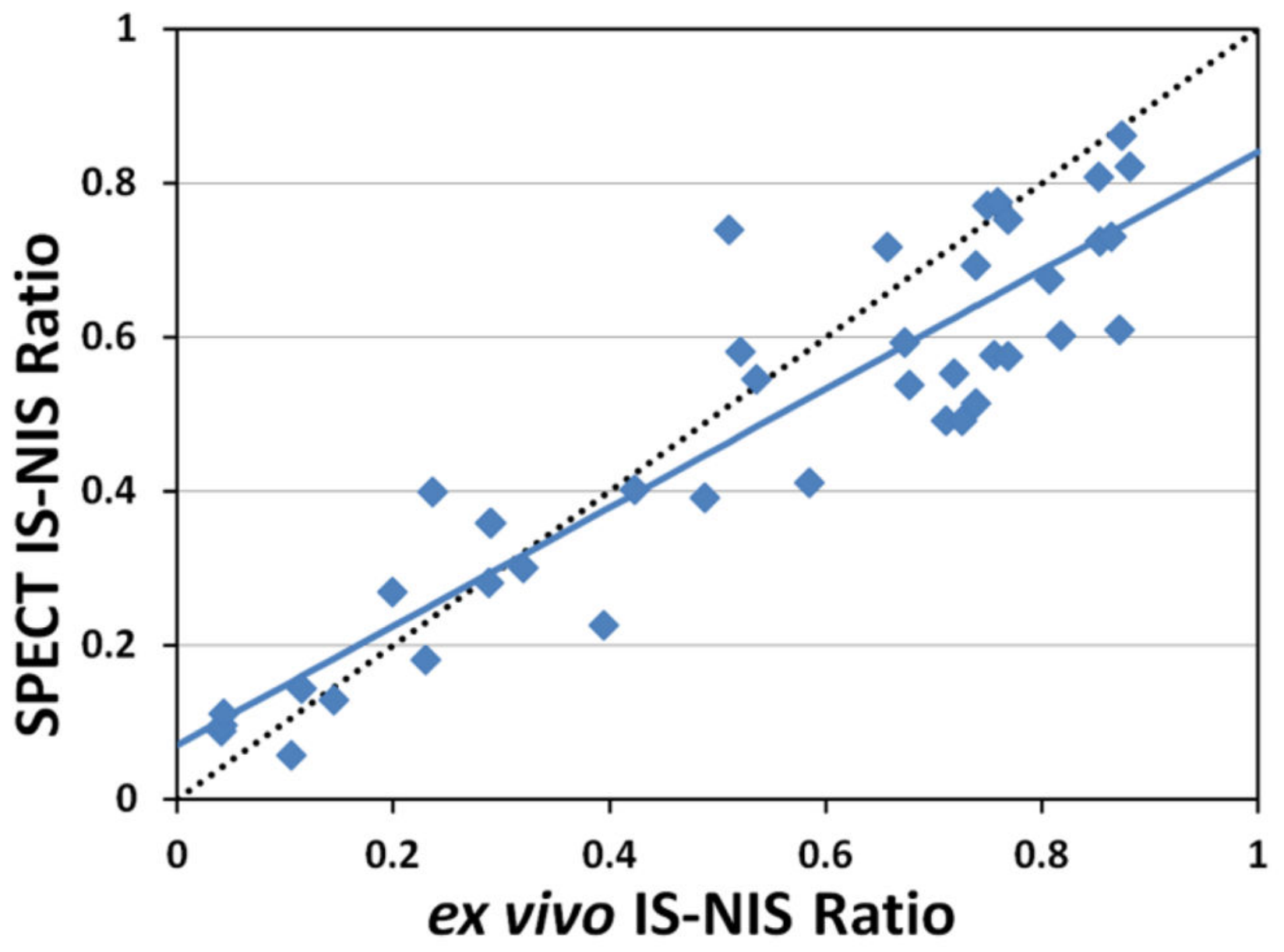

Figure 4.

The ischemic-nonischemic activity concentration ratio between ischemic region and the normal region computed from ${ }^{99 \mathrm{~m}} \mathrm{Tc}$-Sestamibi stress SPECT data as a function of ratio of the corresponding ex vivo data. 


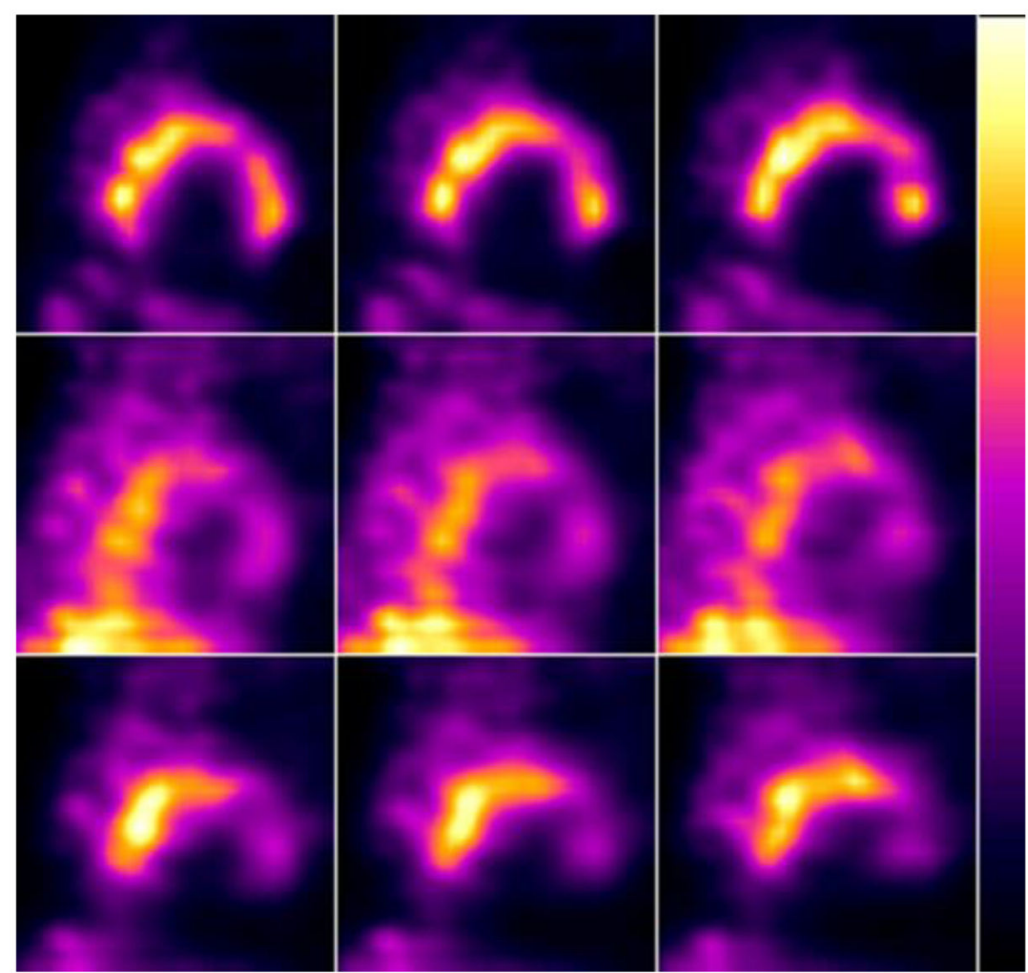

Figure 5.

Short axis slices of the ${ }^{201} \mathrm{Tl}$ rest images from the same dog study as of Figure 2. The top row is from single isotope ${ }^{201} \mathrm{Tl}$ images. The second row is from simultaneously acquired dual isotope data reconstructed without crosstalk compensation. The third row is from simultaneously acquired dual isotope data reconstructed with crosstalk compensation. 


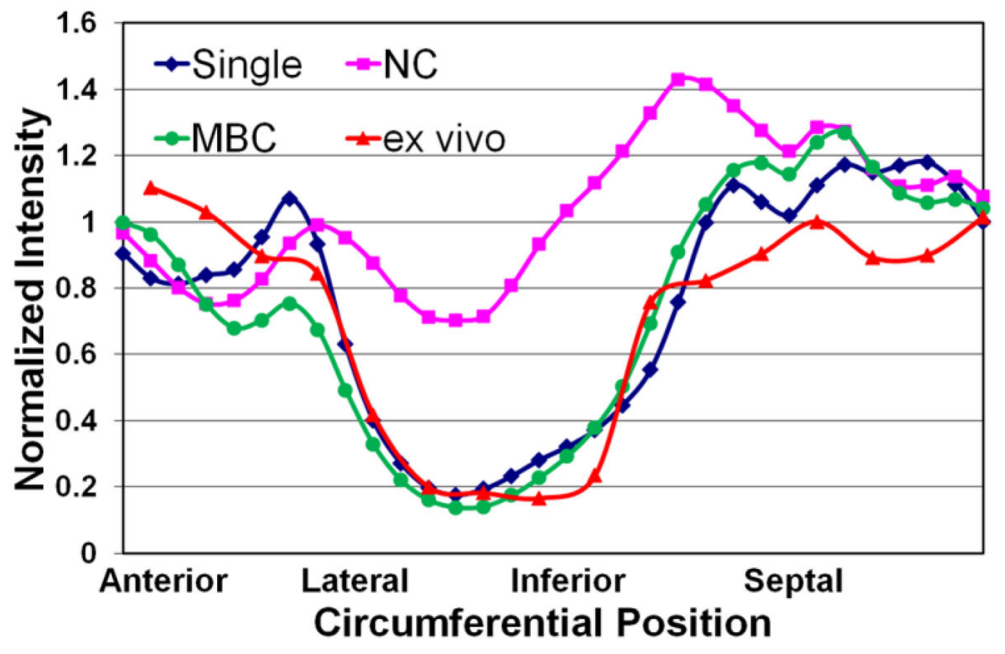

Figure 6.

Circumferential profiles of ${ }^{201} \mathrm{Tl}$ rest images and the ex vivo results. Single, single isotope ${ }^{201} \mathrm{Tl}$ image; $\mathrm{NC}$, simultaneously acquired dual isotope data reconstructed without crosstalk compensation; and MBC, simultaneously acquired dual isotope data reconstructed with crosstalk compensation. The profiles were normalized to have equal average intensity in the septal-anterior region, which had normal uptake. 

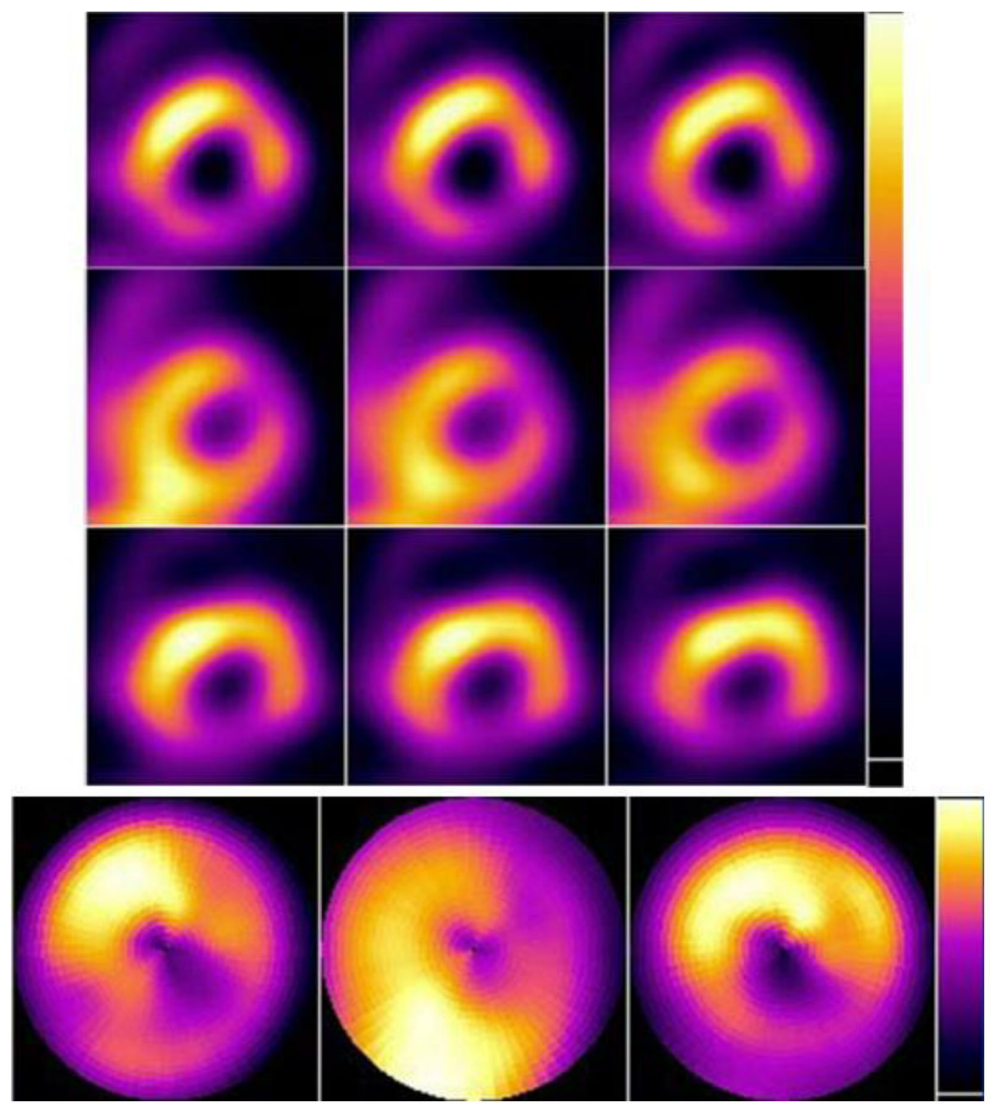

Figure 7.

Short axis slices and the polar maps of the ${ }^{201} \mathrm{Tl}$ rest images from a dog with LCx defect. The top row is from single isotope ${ }^{201} \mathrm{Tl}$ images (single). The second row is from simultaneously acquired dual isotope data reconstructed without crosstalk compensation (NC). The third row is from simultaneously acquired dual isotope data reconstructed with crosstalk compensation (MBC). From left to right, the polar maps are for single, NC, and $\mathrm{MBC}$, respectively. 


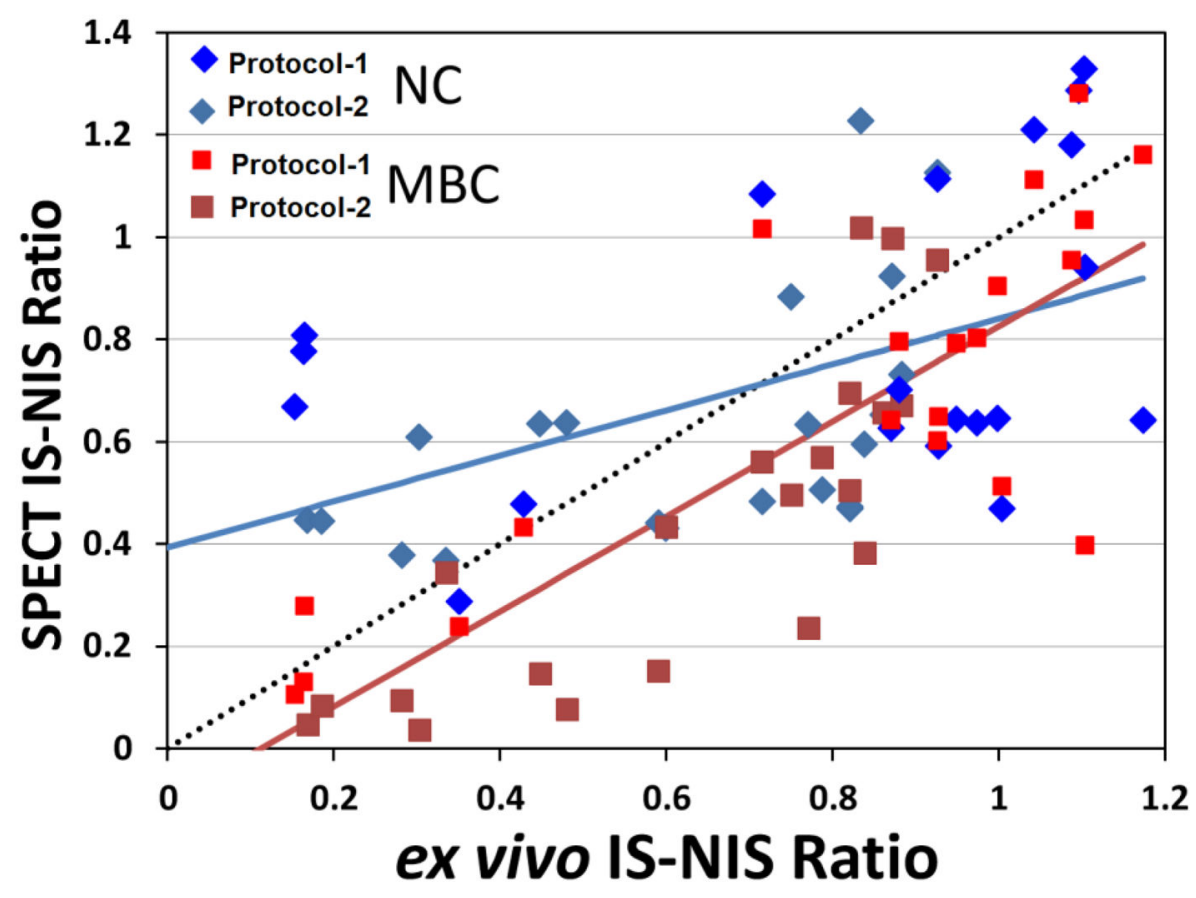

Figure 8.

The activity concentration ratio between the ischemic region and non-ischemic region computed from ${ }^{201} \mathrm{Tl}$ rest SPECT data plotted as a function of the ratio from the ex vivo counting data from all 50 dogs. $\mathrm{NC}$, simultaneously acquired dual isotope data reconstructed without crosstalk compensation; and MBC, simultaneously acquired dual isotope data reconstructed with crosstalk compensation. 


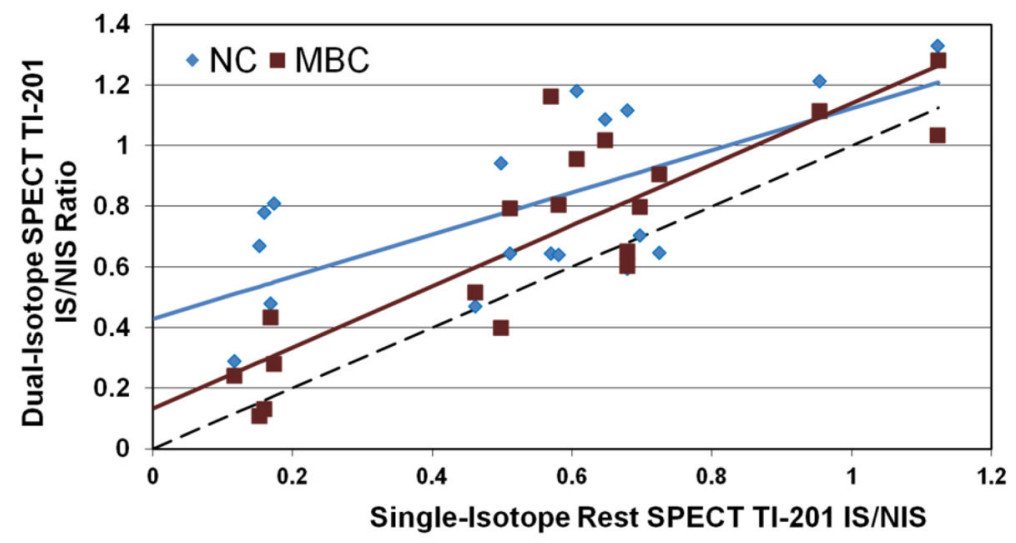

Figure 9.

The activity concentration ratio between the ischemic region and non-ischemic region computed from dual-isotope ${ }^{201} \mathrm{Tl}$ rest SPECT data plotted as a function of the ratio from the single isotope ${ }^{201} \mathrm{Tl}$ rest SPECT data from the $25 \mathrm{dogs}$ imaged with the first protocol. $\mathrm{NC}$ denotes simultaneously acquired dual isotope data reconstructed without crosstalk compensation; MBC denotes simultaneously acquired dual isotope data reconstructed with crosstalk compensation. 